\title{
Changes in genetic correlations by index selection
}

\author{
Y Itoh* \\ Kyoto University, Department of Animal Science, \\ Faculty of Agriculture, Kyoto 606, Japan
}

(Received 13 August 1990; accepted 5 June 1991)

\begin{abstract}
Summary - A formula expressing changes in genetic variances and covariances by index selection in one generation is derived. Then changes in genetic correlation are discussed in 2 simple cases using that formula. When 2 traits involved in the index have equal heritabilities and equal weights, the change in the genetic correlation is always negative and generally large. When selection is on one trait, the genetic correlation with another trait after selection is inclined toward zero.
\end{abstract}

selection index / genetic correlation / Bulmer effect

Résumé - Changements des corrélations génétiques dûs à la sélection sur indice. Une formule est établie pour exprimer les changements des variances et covariances génétiques dûs à la sélection sur indice. Les changements des corrélations génétiques sont ensuite discutés dans 2 situations simples, à l'aide de cette formule. Quand les 2 caractères de l'indice ont des héritabilités et des coefficients de pondération égaux, le changement de la corrélation génétique est toujours négatif et généralement important. Quand la sélection se fait sur un caractère, les corrélations génétiques avec les autres caractères tendent à se rapprocher de zéro.

indice de sélection / corrélation génétique / effet Bulmer

\section{INTRODUCTION}

Assuming a trait influenced by many loci, Bulmer (1971) showed that a substantial change in additive genetic variance due to selection is caused by gamete phase disequilibrium and derived a formula for the disequilibrium component of the genetic variance. Tallis (1987) proposed an alternative procedure which obtained the same result as that of Bulmer (1971), and extended it to be applicable to multiple traits. Furthermore, Tallis and Leppard (1988) studied the joint effects of index selection and assortative mating on multiple traits. Index selection affects

* Present address : Development Department, Nihon Schering KK, Nishimiyahara 2-664, Yodogawa-ku, Osaka 532, Japan. 
various genetic parameters. Especially changes in genetic correlations are important in multiple-trait selection. The objectives of this note are to show explicitly the formula expressing the changes in genetic variances and covariances due to index selection in one generation based on the results of Tallis (1987) and Tallis and Leppard (1988), and to discuss the changes in genetic correlations in some simple cases based on that formula.

\section{A GENERAL FORMULA FOR CHANGES IN VARIANCES AND COVARIANCES}

Let $\mathbf{P}$ and $\mathbf{G}$ be phenotypic and genetic variance-covariance matrices, respectively, and let $\mathbf{b}$ be a vector of index weights. If the variance of a selection index, denoted by $\sigma_{I}^{2}=\mathbf{b}^{\prime} \mathbf{P b}$, is changed by selection to be $\sigma_{I S}^{2}=(1+k) \sigma_{I}^{2}$, then $\mathbf{P}$ becomes :

$$
\mathbf{P}_{S}=\mathbf{P}+\frac{k}{\mathbf{b}^{\prime} \mathbf{P b}} \mathbf{P b b}^{\prime} \mathbf{P}
$$

which can be derived from eq 6 of Tallis and Leppard (1988). This result holds without assuming a normal distribution. If a normal distribution and truncation selection are assumed, the value of $k$ is determined only by a selection rate. Then, from Robertson (1966) or Bulmer (1980, p 163, Eq 9.29), $k$ is expressed as :

$$
k=-i(i-x)
$$

where $x$ and $i$ are respectively the abscissa at the truncation point and the mean of the selected population in the standard normal distribution, that is, $i$ is the selection intensity. The formula obtained by substituting (2) into (1) becomes identical to Eq 10 of Tallis (1965) which was obtained by assuming normality completely. Because $i>0$ and $i<x$ in truncation selection, $k<0$. And from $\sigma_{I S}^{2}>0, k>-1$. Thus, the possible range of $k$ is :

$$
-1<k<0 .
$$

This inequality will be used in the succeeding sections. The value of $k$ for various selection rates can be calculated from a table on the normal distribution ( $e g$ Pearson, 1931, table II) as shown in table I.

Now consider cases where selection intensities and index weights are different in 2 sexes, and let us denote $\mathbf{b}$ and $k$ in the $j$ th $\operatorname{sex}(j=1,2)$ by $\mathbf{b}_{j}$ and $k_{j}$, respectively. When selection changes $\mathbf{P}$ to $\mathbf{P}_{S j}=\left(\mathbf{I}+\mathbf{K}_{j}\right) \mathbf{P}$ in the $j$ th sex, it can be shown that $\mathbf{G}$ in the next generation after selection becomes :

$$
\mathbf{G}_{S}=\mathbf{G}+(1 / 4) \mathbf{G} \mathbf{P}^{-1}\left(\mathbf{K}_{\mathbf{1}}+\mathbf{K}_{2}\right) \mathbf{G}
$$

from the result of Tallis (1987). In index selection, from (1), $\mathbf{K}_{j}$ is expressed as :

$$
\mathbf{K}_{j}=\left(\mathbf{P}_{S j}-\mathbf{P}\right) \mathbf{P}^{-1}=\frac{k_{j}}{\mathbf{b}_{j}^{\prime} \mathbf{P} \mathbf{b}_{j}} \mathbf{P b}_{j} \mathbf{b}_{j}^{\prime} .
$$


Table I. The abscissa at the truncation point of the standard normal distribution $(x)$, the selection intensity $(i)$ and the value of $k=-i(i-x)$ for various selection rates $(p)$.

\begin{tabular}{lccc}
\hline \multicolumn{1}{c}{$p$} & $x$ & $i$ & $k$ \\
\hline 0.001 & 3.0902 & 3.3671 & -0.9322 \\
0.01 & 2.3263 & 2.6652 & -0.9032 \\
0.1 & 1.2816 & 1.7550 & -0.8309 \\
0.2 & 0.8416 & 1.3998 & -0.7814 \\
0.3 & 0.5244 & 1.1590 & -0.7355 \\
0.4 & 0.2533 & 0.9659 & -0.6882 \\
0.5 & 0.0 & 0.7979 & -0.6366 \\
0.6 & -0.2533 & 0.6439 & -0.5777 \\
0.7 & -0.5244 & 0.4967 & -0.5072 \\
0.8 & -0.8416 & 0.3500 & -0.4170 \\
0.9 & -1.2816 & 0.1950 & -0.2879 \\
0.99 & -2.3263 & 0.0269 & -0.0634 \\
0.999 & -3.0902 & 0.0034 & -0.0104 \\
\hline
\end{tabular}

Substituting this into (3), we obtain :

$$
\mathbf{G}_{S}=\mathbf{G}+\frac{1}{4} \mathbf{G}\left(\frac{k_{1}}{\mathbf{b}_{1}^{\prime} \mathbf{P} \mathbf{b}_{1}} \mathbf{b}_{1} \mathbf{b}_{1}^{\prime}+\frac{k_{2}}{\mathbf{b}_{2}^{\prime} \mathbf{P} \mathbf{b}_{2}} \mathbf{b}_{2} \mathbf{b}_{2}^{\prime}\right) \mathbf{G}
$$

It can be shown that the diagonal elements of the latter term of the right hand side of (5) are always negative. It follows that the additive genetic variances, as well as heritabilities, always decrease by index selection irrespective of the values of genetic parameters and index weights. When the same index weights are used in both sexes, (5) reduces to :

$$
\mathbf{G}_{S}=\mathbf{G}+\frac{\bar{k}}{2 \mathbf{b}^{\prime} \mathbf{P b}} \mathbf{G b b}^{\prime} \mathbf{G}
$$

where $\bar{k}=\left(k_{1}+k_{2}\right) / 2$ and $\mathbf{b}$ is a common vector of index weights. This equation will be used in the following sections to derive changes in genetic correlations.

The change in $\mathbf{G}$ in only one generation of selection has been described above. But this change is transitory. If selection is not practiced in the next generation, this change is halved and $\mathbf{G}$ goes back toward its original value. When index selection is repeated for many generations, $\mathbf{G}$ and $\mathbf{P}$ continue to change until equilibrium is attained. The values of $\mathbf{G}$ and $\mathbf{P}$ in each generation, as well as in equilibrium, can be computed iteratively if Eq 24 of Tallis (1987) is used with $\mathbf{K}_{j}$ in (4). Then $k_{j}$ in each generation needs to be known. However, the distribution of a population after selection is no longer normal, so $k_{j}$ cannot be determined precisely. If we assume normality throughout, we can compute $\mathbf{G}$ and $\mathbf{P}$ in equilibrium. But we do not know whether this approximation is appropriate or not. Therefore, we will discuss mainly the parameter values after only 1 generation of selection and show the equilibrium values only as references. In the calculation of the equilibrium values, 
the changes of genetic variances and covariances are of course taken into account, but environmental variances and covariances are assumed to remain constant. When genetic parameters are changed, generally index weights should be recalculated in each generation. However, in the following sections, only simple cases are discussed in which index weights can be assumed to be constant.

\section{EXAMPLE 1 : TWO TRAITS WITH EQUAL HERITABILITIES AND EQUAL WEIGHTS}

First we consider the simplest index selection with 2 traits which have equal heritabilities and equal index weights in both sexes. The traits are assumed to be standardized to have unit phenotypic variances for simplicity. Then, $\mathbf{P}$ and $\mathbf{G}$ before selection and $\mathrm{b}$ are :

$$
\mathbf{P}=\left[\begin{array}{cc}
1 & r_{P} \\
r_{P} & 1
\end{array}\right], \quad \mathbf{G}=h^{2}\left[\begin{array}{cc}
1 & r_{G} \\
r_{G} & 1
\end{array}\right], \quad \mathbf{b}=\left[\begin{array}{l}
1 \\
1
\end{array}\right]
$$

where $r_{P}$ and $r_{G}$ are the phenotypic and genetic correlations and $h^{2}$ is the heritability. Substituting these matrices and vector into (6), we obtain :

$$
\mathbf{G}_{S}=\mathbf{G}+\frac{\bar{k} h^{4}\left(1+r_{G}\right)^{2}}{4\left(1+r_{P}\right)}\left[\begin{array}{ll}
1 & 1 \\
1 & 1
\end{array}\right] .
$$

Thus, the genetic correlation in the next generation after selection becomes :

$$
r_{G S}=\frac{h^{2} r_{G}+\bar{k} h^{4}\left(1+r_{G}\right)^{2} / 4\left(1+r_{P}\right)}{h^{2}+\bar{k} h^{4}\left(1+r_{G}\right)^{2} / 4\left(1+r_{P}\right)}=\frac{4\left(1+r_{P}\right) r_{G}+\bar{k} h^{2}\left(1+r_{G}\right)^{2}}{4\left(1+r_{P}\right)+\bar{k} h^{2}\left(1+r_{G}\right)^{2}} .
$$

The change in the genetic correlation is :

$$
\Delta r_{G}=r_{G S}-r_{G}=\frac{\bar{k} h^{2}\left(1-r_{G}\right)\left(1+r_{G}\right)^{2}}{4\left(1+r_{P}\right)+\bar{k} h^{2}\left(1+r_{G}\right)^{2}} .
$$

It is obvious that the numerator of (8) is always negative. On the other hand, the fact that the denominator of $(8)$ is always positive can be proved in the following way. Because the environmental variance-covariance matrix, $\mathbf{P}-\mathbf{G}$, is positive definite, its characteristic roots should all be positive. It can be shown that the characteristic roots of $\mathbf{P}-\mathbf{G}$ in this example are :

$$
\lambda_{1}=1+r_{P}-h^{2}\left(1+r_{G}\right) \text { and } \lambda_{2}=1-r_{P}-h^{2}\left(1-r_{G}\right) .
$$

From $\lambda_{1}>0$, we obtain :

$$
1+r_{P}>h^{2}\left(1+r_{G}\right) .
$$

Using this inequality, the denominator of $(8)$ becomes :

$$
\begin{aligned}
4\left(1+r_{P}\right)+\bar{k} h^{2}\left(1+r_{G}\right)^{2} & >4 h^{2}\left(1+r_{G}\right)+\vec{k} h^{2}\left(1+r_{G}\right)^{2} \\
& =h^{2}\left(1+r_{G}\right)\left\{4+\bar{k}\left(1+r_{G}\right)\right\}>0 .
\end{aligned}
$$


Thus, it has been proved that always $\Delta r_{G}<0$, that is, $r_{G S}<r_{G}$. Therefore, the genetic correlation after selection is inclined toward -1 as compared with the genetic correlation before selection. This effect of the selection is undesirable for the selection in the next generation.

Example values of $\Delta r_{G}$ for some $r_{G}$ and $r_{P}$ are shown in table II where we assume that $h^{2}=0.5$ and the selection rates are 0.1 in males and 0.5 in females. From table I, it is found that $\bar{k}=-0.7337$ approximately in this case. Table II contains only the combinations of $r_{P}$ and $r_{G}$ that satisfy the condition that $\lambda_{1}>0$ and $\lambda_{2}>0$ where $\lambda_{1}$ and $\lambda_{2}$ are defined in (9). This table shows that always $\Delta r_{G}<0$ as stated above and that the change in $r_{G}$ is generally large in spite of the moderate selection rates and only one generation of selection. Table III shows the difference, denoted by $\Delta r_{G}^{*}$, between the initial value of $r_{G}$ and its equilibrium value attained after repeated selections for many generations on the same condition as in table II. Although the value of $\Delta r_{G}^{*}$ in table III is approximate, it is obvious that $\Delta r_{G}^{*}$ has the same tendency as $\Delta r_{G}$ and its absolute value is generally very large. From these facts, we conclude that the genetic correlation could be changed easily in undesirable direction by index selection.

Table II. The change in the genetic correlation $\left(\Delta_{r_{G}^{*}}\right)$ by index selection of 1 generation with 2 traits which the same heritability, 0.5 , and the same index weights where the selection rates are 0.1 in males and 0.5 in females.

\begin{tabular}{rccccccccc}
\hline & \multicolumn{7}{c}{$r_{P}$} \\
\cline { 2 - 9 }$r_{G}$ & -0.7 & -0.5 & -0.3 & -0.1 & 0.0 & 0.1 & 0.3 & 0.5 & 0.7 \\
\hline & $\Delta_{r_{G}^{*}}$ & & & & & & & & \\
-0.7 & -0.048 & -0.029 & -0.020 & -0.016 & -0.014 & -0.013 & & & \\
-0.5 & -0.124 & -0.072 & -0.051 & -0.039 & -0.035 & -0.032 & & & \\
-0.3 & & -0.128 & -0.089 & -0.068 & -0.061 & -0.055 & -0.047 & & \\
-0.1 & -0.192 & -0.131 & -0.099 & -0.088 & -0.080 & -0.067 & & \\
0.0 & & -0.151 & -0.113 & -0.101 & -0.091 & -0.076 & -0.065 & \\
0.1 & & -0.170 & -0.127 & -0.112 & -0.101 & -0.084 & -0.072 & \\
0.3 & & & -0.199 & -0.146 & -0.128 & -0.115 & -0.095 & -0.081 & \\
0.5 & & & -0.149 & -0.130 & -0.115 & -0.094 & -0.080 & -0.069 \\
0.7 & & & & -0.125 & -0.108 & -0.095 & -0.077 & -0.064 & -0.055 \\
\hline
\end{tabular}

Here we comment briefly on a case where 2 traits have antagonistic weights. Let us put :

$$
\mathbf{b}^{\prime}=\left[\begin{array}{ll}
1 & -1
\end{array}\right]
$$

and use the same $\mathbf{P}$ and $\mathbf{G}$ as in (7). Then, in the same way as the above example, the change in the genetic correlation by the selection using this antagonistic weights can be shown to be :

$$
\Delta r_{G}=-\frac{\bar{k} h^{2}\left(1+r_{G}\right)\left(1-r_{G}\right)^{2}}{4\left(1-r_{P}\right)+\bar{k} h^{2}\left(1-r_{G}\right)^{2}} .
$$


Table III. The difference $\left(\Delta_{r_{G}^{*}}\right)$ between the initial value of genetic correlation and its equilibrium value when index selection is practiced on the same condition as in table II.

\begin{tabular}{rccccccccc}
\hline & \multicolumn{10}{c}{$r_{P}$} \\
\cline { 2 - 10 }$r_{G}$ & -0.7 & -0.5 & -0.3 & -0.1 & 0.0 & 0.1 & 0.3 & 0.5 & 0.7 \\
\hline & $\Delta_{r_{G}^{*}}$ & & & & & & & & \\
-0.7 & -0.064 & -0.042 & -0.032 & -0.026 & -0.024 & -0.022 & & & \\
-0.5 & -0.148 & -0.096 & -0.073 & -0.060 & -0.055 & -0.050 & & & \\
-0.3 & & -0.159 & -0.120 & -0.097 & -0.089 & -0.082 & -0.072 & & \\
-0.1 & -0.229 & -0.166 & -0.134 & -0.122 & -0.113 & -0.098 & & \\
0.0 & & -0.188 & -0.150 & -0.137 & -0.126 & -0.109 & -0.097 & \\
0.1 & & & -0.208 & -0.164 & -0.149 & -0.137 & -0.119 & -0.105 & \\
0.3 & & & -0.241 & -0.183 & -0.165 & -0.151 & -0.130 & -0.114 & \\
0.5 & & & -0.184 & -0.164 & -0.148 & -0.126 & -0.110 & -0.098 \\
0.7 & & & & -0.155 & -0.135 & -0.120 & -0.101 & -0.087 & -0.077 \\
\hline
\end{tabular}

This equation can also be obtained from (8) by affixing minus signs to all of $r_{G}, r_{P}$ and $\Delta r_{G}$. Using $\lambda_{2}>0$ in (9), it can be shown that $\Delta r_{G}$ in (10) is always positive. Thus, in this case with the antagonistic weights, the completely reverse results are obtained in comparison with the above case with equal weights. However, in both cases, the genetic correlation is changed in undesirable direction. If one wants the numerical examples of $\Delta r_{G}$ and $\Delta r_{G}^{*}$ using the antagonistic weights, they can be obtained from tables II and III reverting all the signs of the value of $r_{G}, r_{P}, \Delta r_{G}$ and $\Delta r_{G}^{*}$.

\section{EXAMPLE 2 : SELECTION ON ONE OF TWO TRAITS}

Next, we consider 2 traits again, but selection is based on only one trait. The traits are assumed to be standardized to have unit phenotypic variances as in Example 1. Then, $\mathbf{P}$ and $\mathbf{G}$ before selection and $\mathbf{b}$ are :

$$
\mathbf{P}=\left[\begin{array}{cc}
1 & r_{P} \\
r_{P} & 1
\end{array}\right], \quad \mathbf{G}=\left[\begin{array}{cc}
h_{1}^{2} & h_{1} h_{2} r_{G} \\
h_{1} h_{2} r_{G} & h_{2}^{2}
\end{array}\right], \quad \mathbf{b}=\left[\begin{array}{l}
1 \\
0
\end{array}\right]
$$

where $h_{1}^{2}$ and $h_{2}^{2}$ are the heritabilities of the 2 traits and $r_{P}$ and $r_{G}$ are as in Example 1. From (6), the genetic variance-covariance matrix in the next generation after selection is :

$$
\mathbf{G}_{S}=\mathbf{G}+\bar{k} h_{1}^{2} / 2\left[\begin{array}{cc}
h_{1}^{2} & h_{1} h_{2} r_{G} \\
h_{1} h_{2} r_{G} & h_{2}^{2} r_{G}^{2}
\end{array}\right] .
$$

Thus, the genetic correlation becomes :

$$
r_{G S}=\frac{h_{1} h_{2} r_{G}\left(1+\bar{k} h_{1}^{2} / 2\right)}{\sqrt{h_{1}^{2}\left(1+\bar{k} h_{1}^{2} / 2\right) \cdot h_{2}^{2}\left(1+\bar{k} h_{1}^{2} r_{G}^{2} / 2\right)}}=r_{G} \sqrt{\frac{1+\bar{k} h^{2} / 2}{1+\bar{k} h_{1}^{2} r_{G}^{2} / 2}} .
$$


It is interesting that $r_{G S}$ does not depend on $r_{P}$ at all. From (12), we obtain

$$
r_{G S} / r_{G}=\sqrt{\frac{1+\bar{k} h_{1}^{2} / 2}{1+\bar{k} h_{1}^{2} r_{G}^{2} / 2}} .
$$

The maximum of $r_{G S} / r_{G}$ is attained when $r_{G}= \pm 1$ irrespective of $\bar{k}$ and $h_{1}^{2}$, and its maximum is +1 . On the other hand, its minimum is attained when $r_{G}=0$, then :

$$
r_{G S} / r_{G} \geq \sqrt{1+\bar{k} h_{1}^{2} / 2}>\sqrt{1-h_{1}^{2} / 2} \geq \sqrt{1-1 / 2}=1 / \sqrt{2} .
$$

Therefore, the possible range of $r_{G S} / r_{G}$ is :

$$
1 / \sqrt{2}<r_{G S} / r_{G} \leq 1
$$

This indicates that the sign of a genetic correlation is not changed by this type of selection and the genetic correlation after selection is inclined toward zero.

Example values of $r_{G S}$ and $\Delta r_{G}$ for some $r_{G}$ 's are shown in table IV where we assume that $h^{2}=0.5$ in both traits and that the selection rates are 0.1 in males and 0.5 in females as in table II. Table IV also contains $\Delta r_{G}^{*}$ which is the difference between the initial value of genetic correlation and its equilibrium value. Note that this table does not have the column of $r_{P}$. This is because the results does not depend on $r_{P}$ as described above. From table IV, we find that $r_{G S}$ is inclined toward zero from its original value $r_{G}$ as stated above and that $\Delta r_{G}$ and $\Delta r_{G}^{*}$ are not so large generally as compared with those in table II and III.

Table IV. The genetic correlation after selection $\left(r_{G S}\right)$ and the change $\left(\Delta r_{G}\right)$ from the initial value $\left(r_{G}\right)$ when selection is on one of 2 traits which have the same heritability, 0.5 , where the selection rates are 0.1 in males and 0.5 in females.

\begin{tabular}{rcccc}
\hline$r_{G}$ & $r_{G S}$ & $r_{G S} / r_{G}$ & $\Delta r_{G}$ & $\Delta r_{G}^{*}$ \\
\hline 0.0 & 0.0 & - & 0.0 & 0.0 \\
\pm 0.1 & \pm 0.090 & 0.904 & $\mathrm{~F} 0.010$ & $\mathrm{~F} 0.013$ \\
\pm 0.2 & \pm 0.181 & 0.907 & $\mathrm{~F} 0.019$ & $\mathrm{~F} 0.025$ \\
\pm 0.3 & \pm 0.273 & 0.911 & $\mathrm{~F} 0.027$ & $\mathrm{~F} 0.036$ \\
\pm 0.4 & \pm 0.367 & 0.917 & $\mathrm{~F} 0.033$ & $\mathrm{~F} 0.045$ \\
\pm 0.5 & \pm 0.463 & 0.925 & $\mathrm{~F} 0.037$ & $\mathrm{~F} 0.051$ \\
\pm 0.6 & \pm 0.561 & 0.935 & $\mathrm{~F} 0.039$ & $\mathrm{~F} 0.053$ \\
\pm 0.7 & \pm 0.663 & 0.947 & $\mathrm{~F} 0.037$ & $\mathrm{~F} 0.050$ \\
\pm 0.8 & \pm 0.769 & 0.962 & $\mathrm{~F} 0.031$ & $\mathrm{~F} 0.042$ \\
\pm 0.9 & \pm 0.881 & 0.979 & $\mathrm{~F} 0.019$ & $\mathrm{~F} 0.026$ \\
\hline
\end{tabular}

$\Delta r_{G}^{*}:$ The difference between the initial value of genetic correlation and its equilibrium value.

Changes in $h_{2}^{2}$, denoted by $\Delta h_{2}^{2}$, induced by selection on the first trait can be derived from (11):

$$
\Delta h_{2}^{2}=\frac{h_{2}^{2}+\bar{k} h_{1}^{2} h_{2}^{2} r_{G}^{2} / 2}{1+\bar{k} h_{1}^{2} h_{2}^{2} r_{G}^{2} / 2}-h_{2}^{2}=\frac{\left(1-h_{2}^{2}\right) \bar{k} h_{1}^{2} h_{2}^{2} r_{G}^{2} / 2}{1+\bar{k} h_{1}^{2} h_{2}^{2} r_{G}^{2} / 2} .
$$


It is obvious that $\Delta h_{2}^{2}<0$, so that $h_{2}^{2}$ always decreases irrespective of the values of genetic parameters. This fact holds good not only in this case, but also in every case of index selection. It is only a special example of the general fact that heritabilities always decrease by index selection irrespective of the values of genetic parameters and index weights as stated above in this note.

\section{REFERENCES}

Bulmer MG (1971) The effect of selection on genetic variability. Am Nat 105, 201211

Bulmer MG (1980) The Mathematical Theory of Quantitative Genetics. Clarendon Press, Oxford

Pearson K (1931) Tables for Statisticians and Biometricians, Part II. Cambridge University Press, Cambridge

Robertson A (1966) A mathematical model of the culling process in dairy cattle. Anim Prod 8, 95-108

Tallis MG (1965) Plane truncation in normal populations. J R Stat Soc Ser B 27, 301-307

Tallis MG (1987) Ancestral covariance and the Bulmer effect. Theor Appl Genet $73,815-820$

Tallis MG, Leppard P (1988) The joint effects of selection and assortative mating on multiple polygenic characters. Theor Appl Genet 75, 278-280 